\title{
A Comparative Study of Empirical Mode Decomposition-Based Filtering for Impact Signal
}

\author{
Liwei Zhan and Chengwei $\mathrm{Li}^{*}$ \\ School of Electrical Engineering and Automation, Harbin Institute of Technology, Harbin 150001, China; \\ zhanliwei333@hit.edu.cn \\ * Correspondence: cwlhit@163.com; Tel.: +86-451-8641-5178
}

Academic Editors: Raúl Alcaraz Martínez and Kevin H. Knuth

Received: 14 October 2016; Accepted: 27 December 2016; Published: 29 December 2016

\begin{abstract}
The Complete Ensemble Empirical Mode Decomposition with Adaptive Noise (CEEMDAN) has been used to propose a new method for filtering time series originating from nonlinear systems. The filtering method is based on fuzzy entropy and a new waveform. A new waveform is defined wherein Intrinsic Mode Functions (IMFs) — which are obtained by CEEMDAN algorithm-are firstly sorted in ascending order (the sorted IMFs is symmetric about center point, because at any point, the mean value of the envelope line defined by the local maxima and the local minima is zero), and the energy of the sorted IMFs are calculated, respectively. Finally, the new waveform with axial symmetry can be obtained. The complexity of the new waveform can be quantified by fuzzy entropy. The relevant modes (noisy signal modes and useful signal modes) can be identified by the difference between the fuzzy entropy of the new waveform and the next adjacent new waveform. To evaluate the filter performance, CEEMDAN and sample entropy, Ensemble Empirical Mode Decomposition (EEMD) and fuzzy entropy, and EEMD and sample entropy were used to filter the synthesizing signals with various levels of input signal-to-noise ratio $\left(\mathrm{SNR}_{\text {in }}\right)$. In particular, this approach is successful in filtering impact signal. The results of the filtering are evaluated by a de-trended fluctuation analysis (DFA) algorithm, revised mean square error (RMSE), and revised signal-to-noise ratio (RSNR), respectively. The filtering results of simulated and impact signal show that the filtering method based on CEEMDAN and fuzzy entropy outperforms other signal filtering methods.
\end{abstract}

Keywords: CEEMDAN; fuzzy entropy; DFA; impact signal; filtering

\section{Introduction}

Understanding the real-time characterization of impact events in mechanical systems is important for health monitoring and reliability analysis [1,2]. The instrumented falling weight impact (IFWI) technique really started to develop with the progress of electronic boards able to record short time events $[3,4]$. At present, experimental research for impact tests mainly focuses on the vertical falling weight impact $[5,6]$. However, there are few studies on the oblique impact test. To simulate these events and estimate the real-time characterization of impact events, an experimental rig was designed to measure the real-time signal under different pendulum angle conditions during the impact test.

However, the measured signal often contains noise when the experiment is conducted. The noise behaves as unwanted frequencies and amplitude oscillations. These oscillations corrupt the signal information. Thus, it is very much necessary to develop a method to extract signal information from the noisy signal. At present, there are many filtering methods that fall mainly into two classes: one is a linear filter and the other a non-linear filter. Linear filters such as an average filter [7] and a Winer filter [8] are suitable to filter signal from a stationary system. However, when the signal comes from a non-linear and non-stationary system, a non-linear filter works best for filtering out noise- the wavelet filter is widely used [9]. The signal is split into low and high frequency coefficients 
in the wavelet filter. The filtered signal can be obtained by soft or hard threshold mechanisms [10]. However, the wavelet basis function has to be pre-defined, and this method is not adaptive in essence. Empirical Mode Decomposition (EMD) was introduced by Huang [11]. This algorithm is an alternative method for analyzing data from non-stationary and non-linear systems, and behaves as the filter bank [12]. The signal can be adaptively decomposed into a set of Intrinsic Mode Functions (IMFs) by EMD. The IMFs carry the detailed information of the signal. For noisy signals, the EMD can decompose the noisy signal into noisy signal modes and useful signal (noise-less) modes. The main task based on EMD filtering is to identify the two class modes (noisy and useful signal modes). Recently, Albert et al. [13] and Peng [14] used a correlation-based threshold to identify the relevant modes (noisy signal modes and useful signal modes). However, the strong correlation between the noisy signal and the first mode make it difficult to identify the relevant mode. Boudraa [15] proposed the consecutive mean squared error (CMSE) to find the relevant modes by setting an appropriate threshold method, but the method can be trapped in a local minima in some situations. To void the case, Boudraa [16] introduced a new method to select the relevant mode by the striking similarity between the probability density function (pdf) of the input signal and each IMF. However, this method performs poorly for the filtering signals containing fractional Gaussian noise when the Hurst parameter is close to unity [17]. There are also some filtering algorithms based on EMD [18-20]. However, mode mixing-in which oscillations of different amplitudes are found in a mode, or similar oscillations are encountered in different modes-often occurs in EMD decomposition. This phenomenon prevents the complete extraction of the signal information. To overcome this disadvantage, Wu and Huang [21] introduced the Ensemble Empirical Mode Decomposition (EEMD), which is a method based on the EMD algorithm. The method follows a study of the statistical characteristic of white noise, and adds white noise of a uniform frequency distribution into EMD to avoid mode mixing. However, if the mean square error (MSE) of the added white noise is large, this will lead to a decrease in the signal-to-noise ratio (SNR) and influence the accuracy of the decomposed result. Even if the ensemble size is increased, the decomposed effect cannot improve dramatically, and the computation time is also increased. If the MSE of the added white noise is small, it will decrease the accuracy of the decomposed result, and it is inevitable to bring mode mixing in the low frequency part of IMF obtained by EEMD decomposition. So, EEMD does not completely solve the mode mixing problem. At present, some EEMD-based filtering methods are developed [22,23]. However, the EEMD algorithm introduces new problems. The added white noise is not eliminated fully, and the additional modes may have been produced because of the interaction between original signal and white noise. To resolve these problems, the complete EEMD with adaptive noise (CEEMDAN) was introduced [24]. This method can overcome additional modes, and eliminates the added white noise. At present, the CEEMDAN has been applied in the analysis of laser speckle [25] and short-term wind speed forecasting [26]. However, its use in signal filtering can be found in a few references $[27,28]$.

In this paper, a new filtering method is proposed. First, the original signal is decomposed by CEEMDAN to obtain IMFs. The axial symmetry waveform (new waveform) can be obtained by sorting IMFs and subsequent calculation of the energy of sorted IMFs. Through calculation the fuzzy entropy of the new waveform, the relevant modes (noisy modes and useful signal modes) can be identified. The criterion of the selected mode is the maximum of the difference between adjacent fuzzy entropies. The simulated signal and measured impact signal are used to filter by the proposed filtering method. For simulated signals with different noise levels, filtering with CEEMDAN and sample entropy, EEMD and fuzzy entropy, and EEMD and sample entropy are compared to evaluate the signal filtering performance. For the measured signal, CEEMDAN and fuzzy entropy, wavelet filter, moving averaging filter, and median filter are used to filter, respectively. The filter performance can be evaluated by de-trended fluctuation analysis (DFA) algorithm, revised mean squared error (RMSE), and revised signal-to-noise ratio (RSNR). The filtering result of simulated and measured signals show that the filtering method based on CEEMDAN and fuzzy entropy outperforms the other filtering methods. 


\section{Related Works}

\subsection{Improved Empirical Mode Decomposition}

Since the empirical mode decomposition (EMD) [11] was proposed by Huang, this algorithm has been successfully applied to filter signals. However, mode mixing occurs in the process of EMD decomposition. To overcome this problem, the EEMD [21] was introduced by adding white noise into the signal. However, the algorithm brings a new drawback. The added noise cannot be eliminated fully, and may produce additional spurious modes. To overcome this drawback, the CEEMDAN algorithm [24] is proposed to resolve the residual white noise and the mode mixing problem. The following is the decomposed steps of the CEEMDAN algorithm:

(1) Decompose signal $x(t)+w_{0} \varepsilon^{i}(t)$ to obtain the first mode by using EMD algorithm.

$$
c_{1}(t)=\frac{1}{N} \sum_{i=1}^{N} c_{1}^{i}(t) i \in\{1, \ldots, N\}
$$

where $w_{0}$ is the ratio of the standard deviation of the added white noise, $\varepsilon^{i}(t)$ is the white noise with unit variance under condition of the $i$-th ensemble number, $N$ is the total ensemble number, and $c_{1}^{i}(t)$ is the first mode obtained EMD decomposition under the condition of $i$-th signal: $x(t)+w_{0} \varepsilon^{i}(t)$.

(2) Compute the difference signal

$$
r_{1}(t)=x(t)-c_{1}(t)
$$

(3) Decompose $r_{1}(t)+w_{1} E_{1}\left(\varepsilon^{i}(t)\right)$ to obtain the first mode and define the second mode by

$$
c_{2}(t)=\frac{1}{N} \sum_{i=1}^{N} E_{1}\left(r_{1}(t)+w_{1} E_{1}\left(\varepsilon^{i}(t)\right)\right)
$$

Here, $E_{1}$ and $\varepsilon^{i}(t)$ stand for a function to extract the first IMF decomposed by EMD and the white noise with unit variance, respectively.

(4) For $k=2, \ldots, K$, calculate the $k$-th residue and obtain the first mode. Define the $(k+1)$-th mode as follows:

$$
c_{k+1}(t)=\frac{1}{N} \sum_{i=1}^{N} E_{1}\left(r_{k}(t)+w_{k} E_{k}\left(\varepsilon^{i}(t)\right)\right)
$$

where $E_{k}(\cdot)$ is a function to extract the $k$-th IMF decomposed by EMD.

(5) Repeat step (4) until the residue contains no more than two extremes. The residual mode is then defined as:

$$
\mathrm{R}(t)=x(t)-\sum_{k=1}^{K} c_{k}(t)
$$

Therefore, the signal $x(t)$ can be expressed as follows:

$$
x(t)=\sum_{k=1}^{K} c_{k}(t)+\mathrm{R}(t)
$$

\subsection{Fuzzy Entropy}

In 1948, Shannon introduced entropy into information theory and proposed the concept of information entropy to measure the uncertainty of an event. Afterwards, the concept of entropy was generalized. The approximate entropy (ApEn) [29], sample entropy (SampEn) [30], and fuzzy entropy [31] have been gradually proposed. ApEn and SampEn have the characteristics of needing shorter data, anti-noise, and being resistant to interference compared to Shannon entropy. The similarity 
of two vectors is defined by step function, which has the property of a two-state classifier. If the input sample meets certain conditions, the sample belongs to some class; otherwise, it belongs to another class. However, the noise signal can be decomposed into noisy modes and noise-less modes by CEEMDAN algorithm. The main task is to identify the demarcation point between noisy mode and noise-less mode. However, the demarcation point is fuzzy and sometimes difficult to identify by Shannon entropy, approximate entropy, and sample entropy. Afterwards, fuzzy entropy—defined by using a fuzzy function-is introduced to distinguish the demarcation fuzziness. The fuzzy entropy is introduced to identify fuzziness between the noisy mode and noise-less mode. The fuzzy entropy algorithm was introduced by Chen et al. [31], in which fuzzy sets were introduced to improve the statistical stability. The detailed description for the fuzzy entropy is as follows:

(1) Given a time sequence $\{x(i): 1 \leq i \leq N\}$ ( $N$ is the sample size) to form a sequence segment by choosing $m$ consecutive values in time sequence $x(i)$

$$
X_{i}^{m}=\{x(i), x(i+1), \ldots, x(i+m-1)\}-x_{0}(i)(i=1,2, \ldots, N-m)
$$

The vector $X_{i}^{m}$ represents $m$ consecutive $x(i)$ values but removing the baseline $x_{0}(i)$.

The $x_{0}(i)$ expression is shown as follows:

$$
x_{0}(i)=\frac{1}{m} \sum_{j=0}^{m-1} x(i+j)(i=1,2, \ldots, N-m)
$$

(2) The distance $d\left[X_{i}^{m}, X_{j}^{m}\right]$ between the vector $X_{i}^{m}$ and $X_{j}^{m}$ is defined

$$
\begin{aligned}
d_{i j}^{m} & =d\left[X_{i}^{m}, X_{j}^{m}\right] \\
& =\max _{k \in(0, m-1)}\left\{\left|\left(x(i+k)-x_{0}(i)\right)-\left(x(j+k)-x_{0}(j)\right)\right|\right\}
\end{aligned}
$$

Here, $i, j=1,2, \ldots, N-m, i \neq j$.

(3) The similarity degree $D_{i j}^{m}$ between $X_{i}^{m}$ and $X_{j}^{m}$ is expressed by the fuzzy function $\mu\left(d_{i j}^{m}, n, r\right)$. The expression of similarity $D_{i j}^{m}$ is as follows:

$$
D_{i j}^{m}=\mu\left(d_{i j}^{m}, n, r\right)=e^{-\left(d_{i j}^{m} / r\right)^{n}}
$$

(4) Definition function

$$
\phi^{m}(n, r)=\frac{1}{N-m} \sum_{i=1}^{N-m}\left(\frac{1}{N-m} \sum_{\substack{j=1 \\ j \neq i}}^{N-m} D_{i j}^{m}\right)
$$

(5) For $m+1$ dimension function, repeat steps (1) to (4), and obtain $\phi^{m+1}(n, r)$

$$
\phi^{m+1}(n, r)=\frac{1}{N-m} \sum_{i=1}^{N-m}\left(\frac{1}{N-m} \sum_{\substack{j=1 \\ j \neq i}}^{N-m} D_{i j}^{m+1}\right)
$$


(6) The fuzzy entropy is defined by:

$$
\operatorname{Fuzzy} \operatorname{En}(m, n, r)=\ln \phi^{m}(n, r)-\ln \phi^{m+1}(n, r)
$$

Here, $m$ is the embedding dimension, $n$ is the similarity weight, $r$ is the tolerance threshold, and $N$ is the segment length.

\section{Filtering Method}

\subsection{Identifying the Relevant Mode}

The noisy signal $x(t)$ is decomposed into noisy modes and useful signal (noise-less modes) modes, as follows:

$$
x(t)=\sum_{i=1}^{k-1} I M F_{i}+\sum_{i=k}^{K} I M F_{i}
$$

Here, the noisy modes are the first $(k-1)$ modes, and the remaining are the useful signal modes (except residue). The main task is to identify the $k$ index of this mode to filter signal. Through the further analysis to satisfy the conditions for obtaining IMFs (at any point, the mean value of the envelope line defined by the local maxima and the local minima is zero), it assumes that the symmetric waveform about the center point can be obtained by sorting the IMFs. To identify the relevant modes (noisy modes and useful signal modes) more effectively, the energy of the sorted IMFs are calculated to obtain the axis symmetric waveform. The obtained waveform is defined as the new waveform. For the convenience of calculation, the new waveform is normalized (the normalized new waveform is abbreviated to NNW). The complexity of the normalized new waveform is measured by fuzzy entropy [31]. Through the experiment, it is found that the fuzzy entropy of the NNWs of the noisy modes is around a certain value, and that of useful signal modes is around another certain value. When the difference is done for adjacent fuzzy entropies, and the absolute value is done for the difference, it shows that the difference of adjacent fuzzy entropy for the NNW of noisy modes or useful signal modes is a small change, and that the difference of noisy mode or useful signal mode is larger, and is the maximum among the difference of absolute value. Thus, the index $k$ of mode can be found by this method. After finding the index $k$ of the relevant mode, the first $(k-1)$ modes are forced to be zero (hard threshold value), and the remaining modes are summed to obtain the filtered signal. The filtering steps are as follows:

(1) The noisy signal $x(t)$ is decomposed to obtain $\operatorname{IMF}_{i}(i=1, \ldots, N)$ by EMD or the improved version.

(2) Each IMF is sorted in ascending order, and the energy of the sorted data is calculated and then normalized to obtain a new waveform (the normalized new waveform is defined as NNW).

(3) The complexity of each $\mathrm{NNW}_{i}$ (except residue) is calculated by fuzzy entropy, and the value of fuzzy entropy is marked as $E_{i}(i=1, \ldots, M-1)$. Here, $\mathrm{M}$ is the number of modes by EMD or the improved version.

(4) The difference of adjacent $E_{i}$ is obtained, and the absolute value of the difference is calculated.

$$
D_{j}=\left|E_{j}-E_{j+1}\right|(j=1,2, \ldots, M-2)
$$

(5) The relevant mode is identified.

$$
\mathrm{r}=\operatorname{argmax}\left(D_{j}\right)+1
$$

(6) The filtered signal is obtained.

$$
\widetilde{x}(t)=\sum_{m=r}^{M} I M F_{m}(t)
$$




\subsection{Application}

Now, the original signal is filtered using the proposed filtering method. Signal $x(t)$ comprises two periodic signals of differing frequencies (one frequency $f_{1}$ is $3 \mathrm{~Hz}$, and the other $f_{2}$ is $6 \mathrm{~Hz}$,).

$$
x(t)=\cos \left(2 \pi f_{1} t\right)+\sin \left(2 \pi f_{2} t\right)
$$

Here, the input signal-to-noise ratio $\left(\mathrm{SNR}_{\text {in }}\right)$ is $3 \mathrm{~dB}$, and the length of data is 1024 . The signal $x(t)$ is decomposed into nine modes by CEEMDAN (the ratio of the standard deviation of added white noise is 0.15 , and ensemble number is 80 ). If the signal $x(t)$ is known, the seventh and eighth modes are the useful signal mode, and the other are the noisy modes (except residue). The filtered signal is the sum of last three modes (the seventh, eighth, and ninth modes).

Now, the proposed filtering method is used to filter noisy signal. To evaluate the proposed filtering method, it is tested fifty times to prove the filtering effect statistically. For each filtering, the IMFs are first sorted by ascending order, then the energy of the sorted IMFs are calculated and normalized. Figure 1 shows the IMFs obtained by CEEMDAN, Figure 2 shows the sorted IMFs in ascending order (from the Figure 2, it is found that the sorted IMFs (SIMFs) are symmetrical about the center point), and Figure 3 shows each NNW (except residue) (Figures 1-3 are IMFs, SIMFs, and the NNW obtained by the first test respectively). From Figure 3, it can be seen that the first six NNWs are the waveform corresponding to noisy modes, and the last two NNWs are the waveform corresponding to useful signal modes (noise-less modes). The situation of the remaining 49 tests are similar to the first. The averaged fuzzy entropy of each NNW is in Table 1 for the fifty-run statistical test. The averaged fuzzy entropy of the first six modes is approximately 0.04 , and that of last two modes are around 0.02 . The absolute value of the difference of adjacent fuzzy entropy is shown in Figure 4. The absolute value of the difference of the sixth and seventh NNW is larger than the others in Figure 4. This illustrates that starting with the seventh IMF is the useful mode. The filtered signal is shown in Figure $5 \mathrm{~b}$. This shows that the noisy signal modes and useful signal modes can be effectively identified by the proposed method.
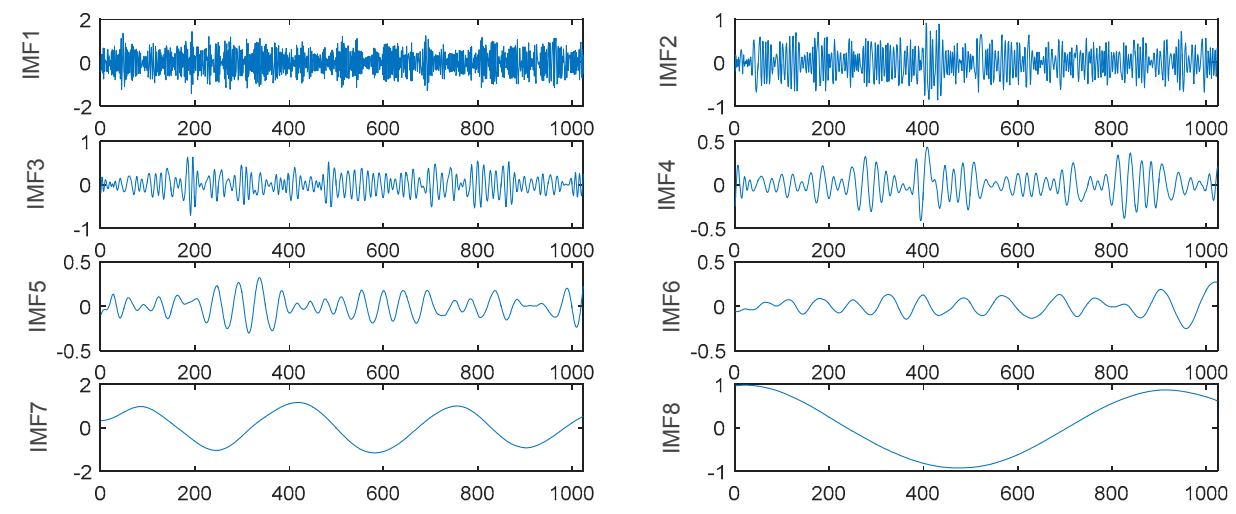

Figure 1. The intrinsic mode functions (IMFs) obtained by the CEEMDAN (complete Ensemble Empirical Mode Decomposition (EEMD) with adaptive noise) algorithm (except residue). 

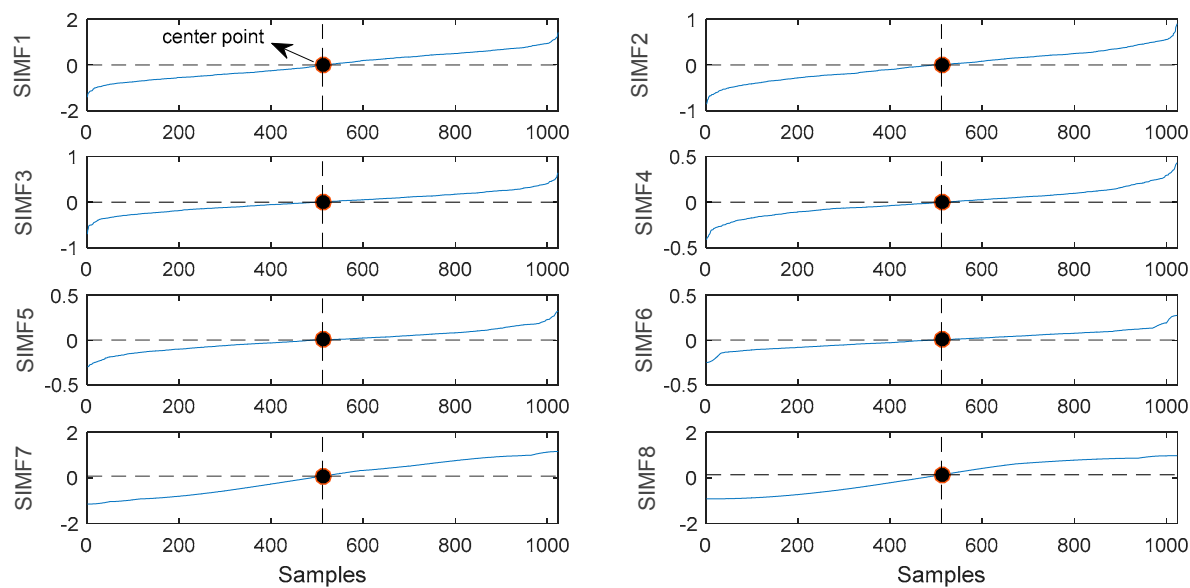

Figure 2. The sorted IMFs (SIMF) of each IMF (except residue).
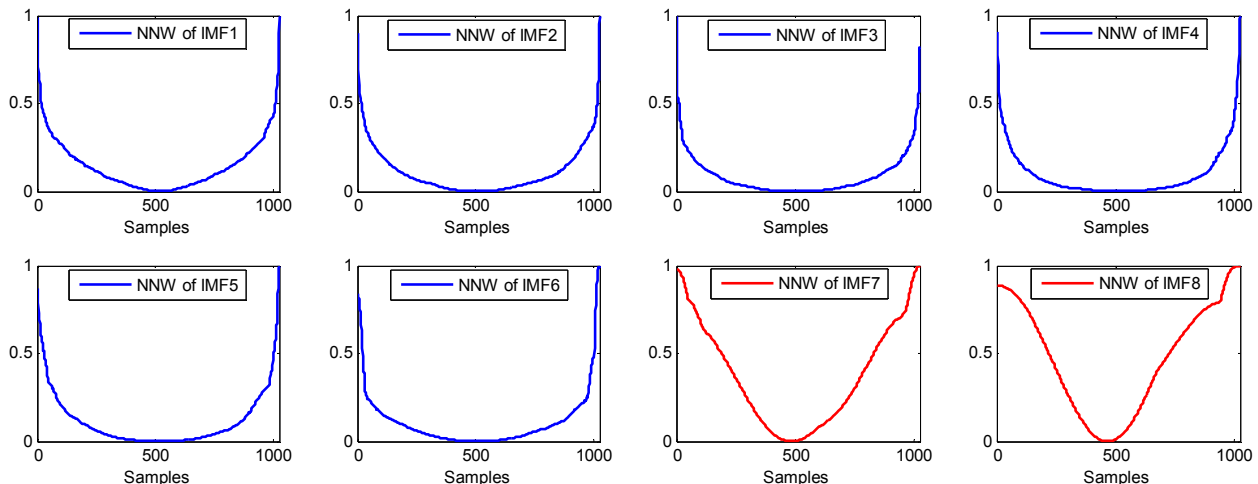

Figure 3. The transformed waveform of each mode (except residue). NNW: normalized new waveform.

Table 1. The averaged fuzzy entropy of each NNW.

\begin{tabular}{ccccccccc}
\hline Changed Waveform & NNW1 & NNW2 & NNW3 & NNW4 & NNW5 & NNW6 & NNW7 & NNW8 \\
\hline Fuzzy Entropy & 0.0438 & 0.0446 & 0.0458 & 0.0469 & 0.0455 & 0.0406 & 0.0246 & 0.0212 \\
\hline
\end{tabular}

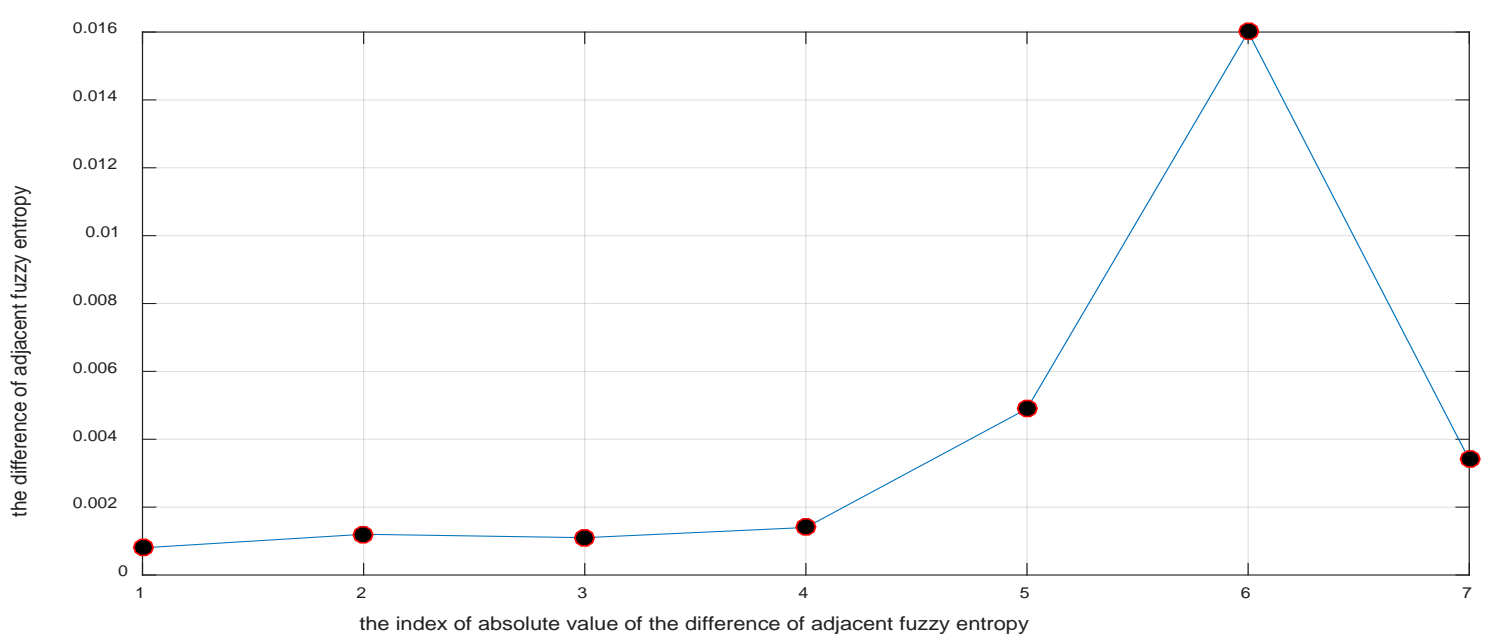

Figure 4. The absolute value of the difference of adjacent fuzzy entropy. 

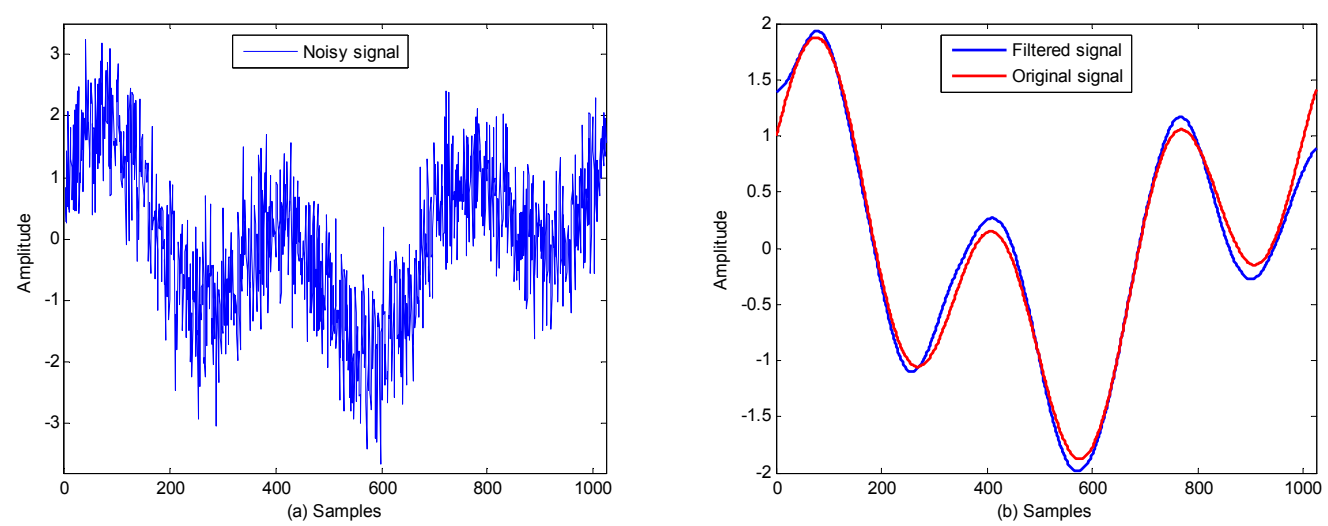

Figure 5. The noisy signal and filtered signal. (a) The noisy signal; (b) The original signal and filtered signal.

\section{Results and Discussion}

In this part, the proposed filtering method is evaluated by two kinds of signal: simulated signal, and measured impact signal.

\subsection{Simulated Signal Filtering}

Here, the five kinds of simulated signal in Figure 6 are used as test signal to filter out noise. To compare the filtered result, the CEEMDAN and fuzzy entropy, CEEMDAN and sample entropy, EEMD and fuzzy entropy, and EEMD and sample entropy are used to filter, and the filtering performance is evaluated at various input signal-to-noise ratios $\left(\mathrm{SNR}_{\mathrm{in}}\right)$. The $\mathrm{SNR}_{\mathrm{in}}$ ranges from $1 \mathrm{~dB}$ to $11 \mathrm{~dB}$ with a fixed step of $2 \mathrm{~dB}$. To quantize the filtering result, the output signal-to-noise ratio $\left(\mathrm{SNR}_{\mathrm{out}}\right)$ and mean square error (MSE) are performed to compare.

$$
\begin{gathered}
\mathrm{SNR}_{\text {out }}=10 \log _{10}\left(\frac{\sum_{n=1}^{N}(y(n))^{2}}{\sum_{n=1}^{N}(\widetilde{y}(n)-y(n))^{2}}\right) \\
\text { MSE }=\frac{1}{N} \sum_{n=1}^{N}(\widetilde{y}(n)-y(n))^{2}
\end{gathered}
$$

where $y(n)$ is the noise-less signal, and $\widetilde{y}(n)$ is the filtered signal.
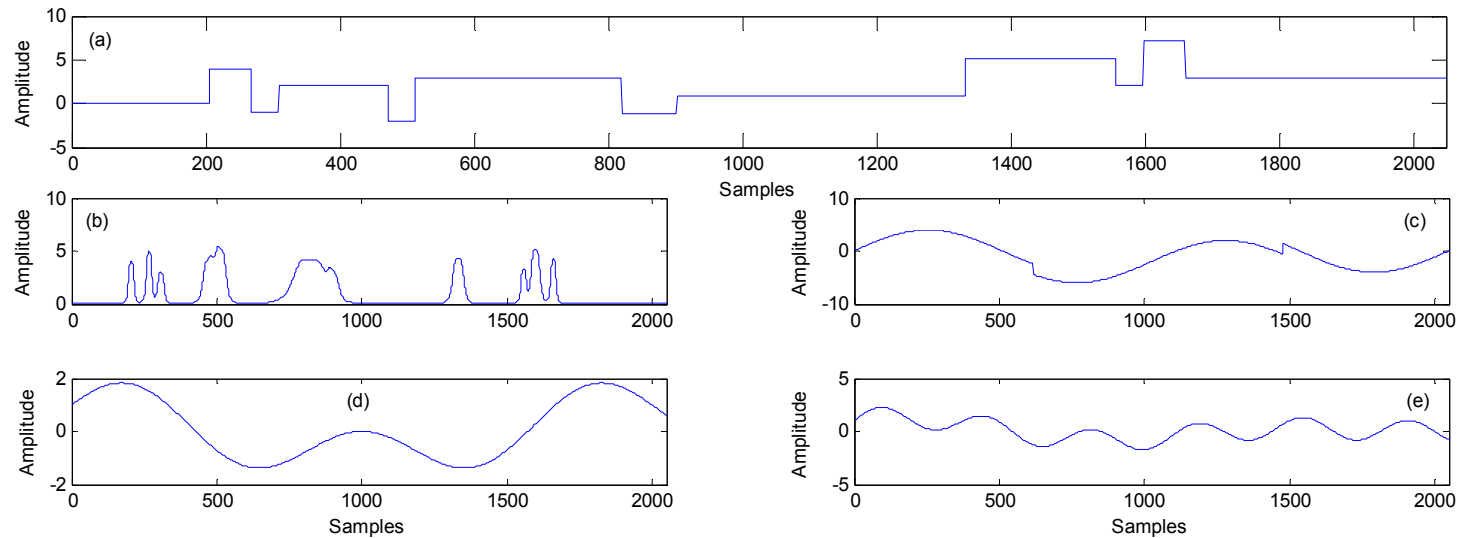

Figure 6. The five kinds of simulated signal. (a) Blocks; (b) Bumps; (c) Heavysine; (d) $y=\cos (2 \pi t)+\sin (5 \pi t) ;(\mathbf{e}) y=\cos (3 \pi t)+\sin (11 \pi t)+\sin (2.5 \pi t)$. 
However, EEMD and CEEMDAN involve added white noise and ensemble number. The obtained IMFs is sensitive to the selection of the parameters related to EEMD and CEEMDAN algorithms. To evaluate the filtering results objectively, the ratio of the standard deviation of the added noise is fixed (0.15). The only change is the ensemble number. For each $\mathrm{SNR}_{\mathrm{in}}$, the different values of ensemble number are set to obtain corresponding $S_{N R}$ out. The statistical results can be obtained by averaging the $\mathrm{SNR}_{\text {out }}$. Here, about a hundred values for ensemble number for each $\mathrm{SNR}_{\text {in }}$ have been tested: that is, values $10-500$ with steps of 5 . Figures 7 and 8 are the statistical SNR out $_{\text {and MSE }}$ for four filtering methods (according to references [31,32] and experimentally, the three parameters $(m, n, r)$ for the fuzzy entropy are $(1,0.2,1)$ and for sample entropy, the parameters $(m, r)$ are $(2,0.15))$.

Figures 7a-e and 8a-e correspond to filtering results of the signal of Table 2. From Figures 7 and 8 , the conclusion is drawn that the $\mathrm{SNR}_{\text {out }}$ of the filtering method based on CEEMDAN and fuzzy entropy is larger than the others, and the MSE of the filtering method based on CEEMDAN and fuzzy entropy is smaller than the others. This shows that the proposed filtering method outperforms the other filtering methods.

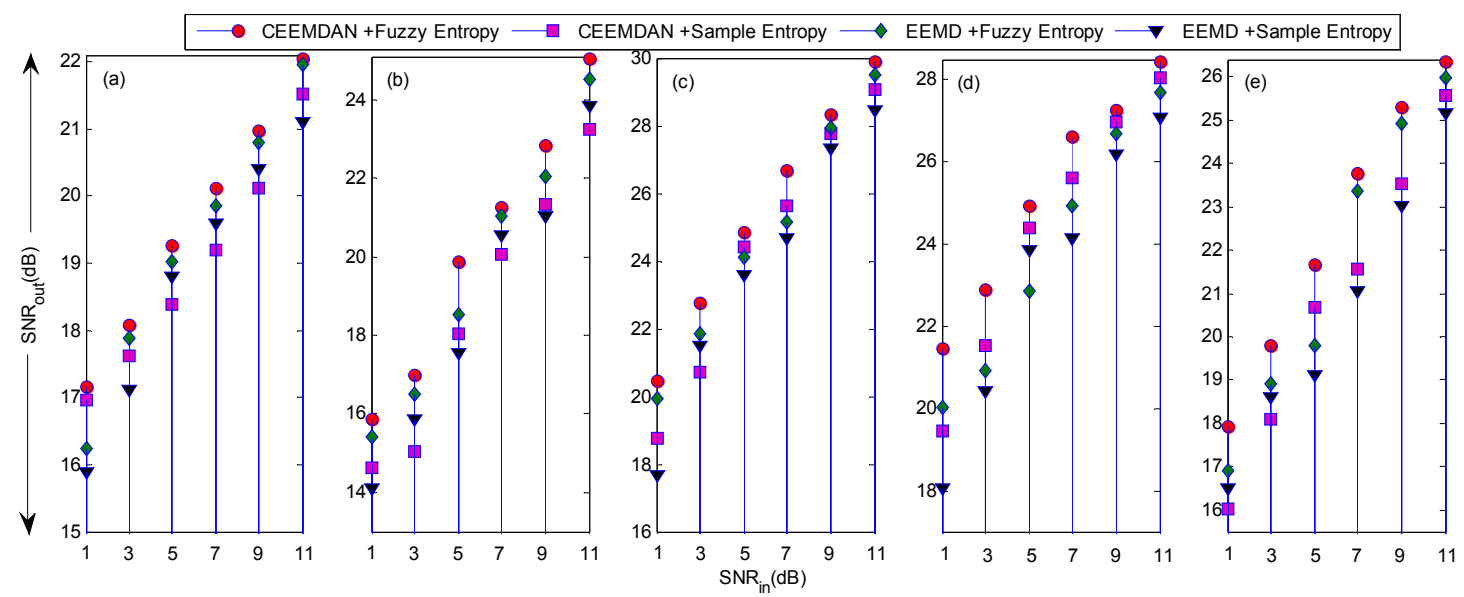

Figure 7. The statistical output signal-to-noise ratio $\left(\mathrm{SNR}_{\mathrm{out}}\right)$ for different simulated signals with various input SNR $\left(\mathrm{SNR}_{\text {in }}\right)$. (a) Blocks; (b) Bumps; (c) Heavysine; (d) $y=\cos (2 \pi t)+\sin (5 \pi t)$; (e) $y=\cos (3 \pi t)+\sin (11 \pi t)+\sin (2 \cdot 5 \pi t)$.

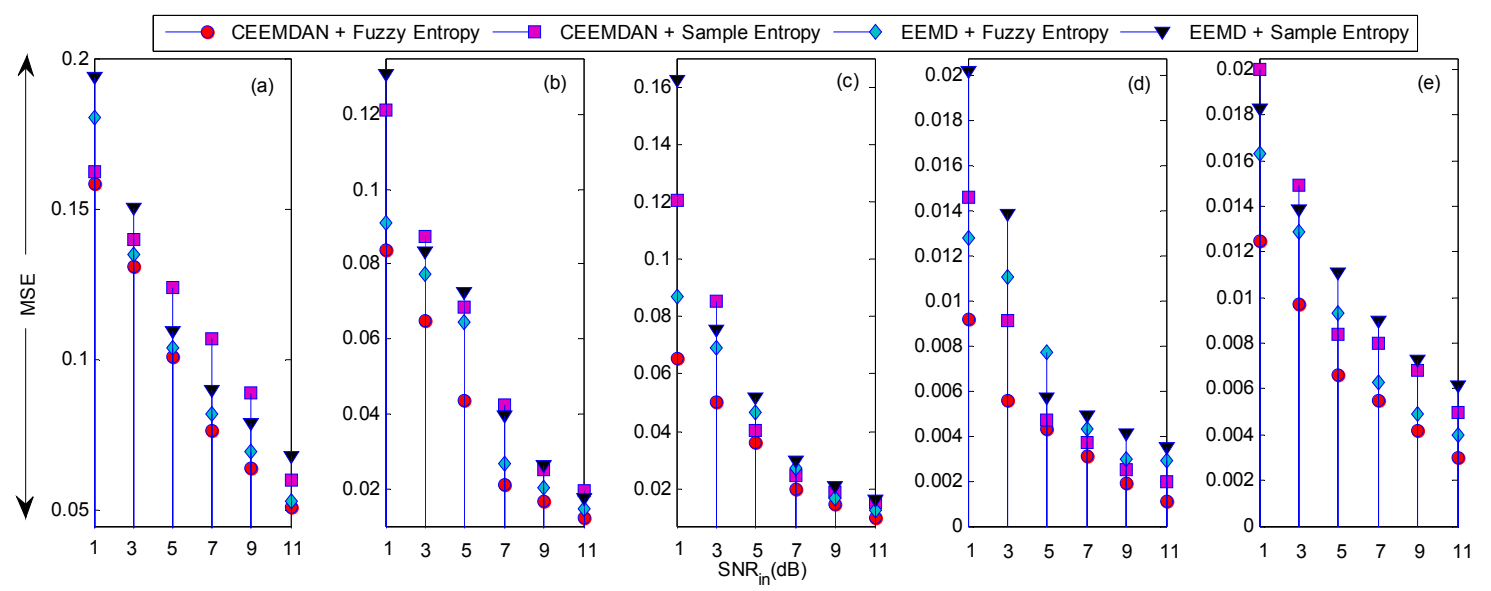

Figure 8. The statistical mean square error (MSE) for different simulated signals with various $\mathrm{SNR}_{\mathrm{in}}$. (a) Blocks; (b) Bumps; (c) Heavysine; (d) $y=\cos (2 \pi t)+\sin (5 \pi t)$; (e) $y=\cos (3 \pi t)+\sin (11 \pi t)+\sin (2.5 \pi t)$. 
Table 2. The five kinds of simulated signal.

\begin{tabular}{ccc}
\hline Order & Signal & Length of Data \\
\hline $\mathrm{a}$ & Blocks & 2048 \\
$\mathrm{~b}$ & Bumps & 2048 \\
$\mathrm{c}$ & Heavysine & 2048 \\
$\mathrm{~d}$ & $y=\cos (2 \pi t)+\sin (5 \pi t)$ & 2048 \\
$\mathrm{e}$ & $y=\cos (3 \pi t)+\sin (11 \pi t)+\sin (2.5 \pi t)$ & 2048 \\
\hline
\end{tabular}

\subsection{Impact Signal Filtering}

In this part, the measurement system is developed to understand the real-time characterization of impact events in mechanical systems. It is important to evaluate health monitoring and reliability analysis. Traditional drop test is generally a vertical drop. To understand characteristics of oblique impact, a novel experiment rig-a swinging rod mechanism—was designed as in Figure 9. The rod can rotate on axis to realize different impact angles. A piezoelectric acceleration sensor was installed as in Figure 9 (the amplitude range of the sensor was $\pm 1000 \mathrm{~g}$, the frequency range was $0.3 \mathrm{KHz}-10 \mathrm{KHz}$, and the sensitivity was $500 \mathrm{mV} / \mathrm{g}$ ). The voltage signal was acquired by an eight channel synchronous data acquisition card, and the sampling rate was $100 \mathrm{KHz}$.

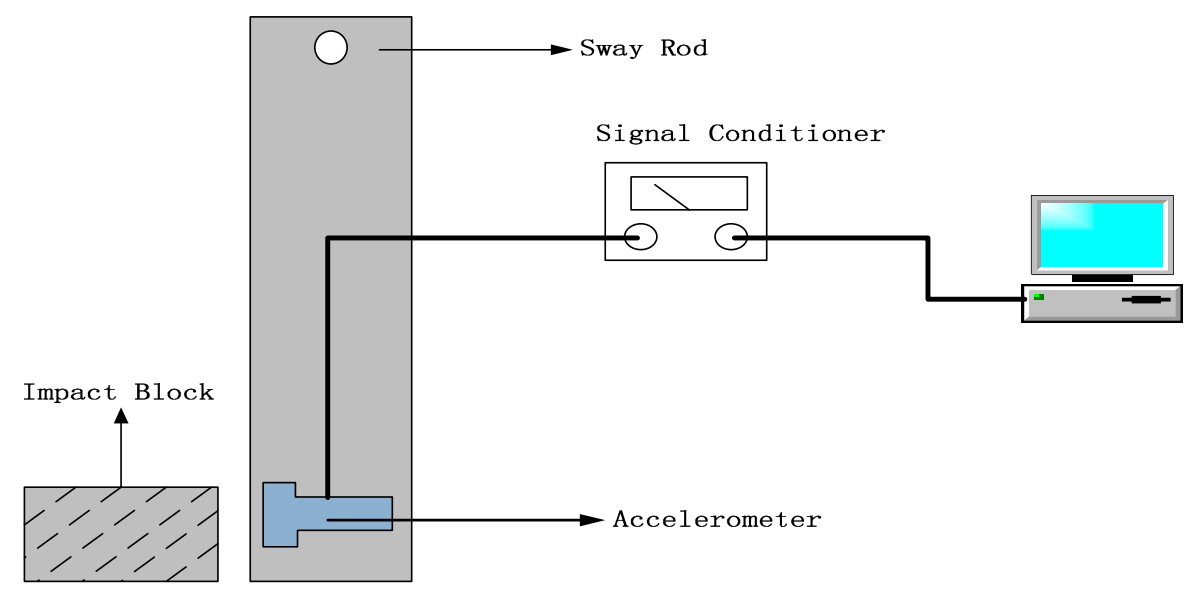

Figure 9. A sketch of the experimental setup.

Now, the experiment was conducted under the condition of a $30^{\circ}$ impact angle. The impact signal was contaminated by coloured noise. The peak value of the impact signal contained the main parameter of physical structure. However, impact time was short, and amplitude of the impact signal changed quickly. Filtering is difficult for impact signals. A traditional bandpass filtering may retrieve the oscillatory component of the signal, but the premise is that the filter parameter is known. The proposed filtering method in this paper can be used to filter noisy signals without knowledge of the signal parameter. The statistical experiment was done for the impact signal by the CEEMDAN and fuzzy entropy under the condition of about a hundred values for ensemble number for: values 10-500 with steps of 5 , and ratio of the standard deviation of the added noise 0.1. The filtering efficiency was compared with proposed filtering method, wavelet filter (the mother wavelet: $\mathrm{db} 10$, and level of decomposition: 8), median filter (the size of the sliding window: 6), and moving average filter (the size of the sliding window: 3) in Figure 10. The run times for the CEEMDAN (the ratio of added white noise is the 0.1, and the ensemble number is ten) and entropy, DWT(Discrete Wavelet Transform), median filter, and moving averaging filters were $7.6377 \mathrm{~s}, 0.9675 \mathrm{~s}, 0.0018 \mathrm{~s}$, and $0.0094 \mathrm{~s}$, respectively (the laptop computer used: i5-3230M, CPU: $2.60 \mathrm{GHz}$, Lenovo, Shen Zhen, China). Figure 10 shows that these methods extract signal information with noticeable ripples and fluctuations. However, the filtered signal based on CEEMDAN and entropy was very smooth and showed signal 
characteristics. From the computing result, the filter based on CEEMDAN required more computing time; however, the filtering effect was the greatest among the four filtering methods, and the computing time for the filter method based on CEEMDAN also met the engineering requirement.
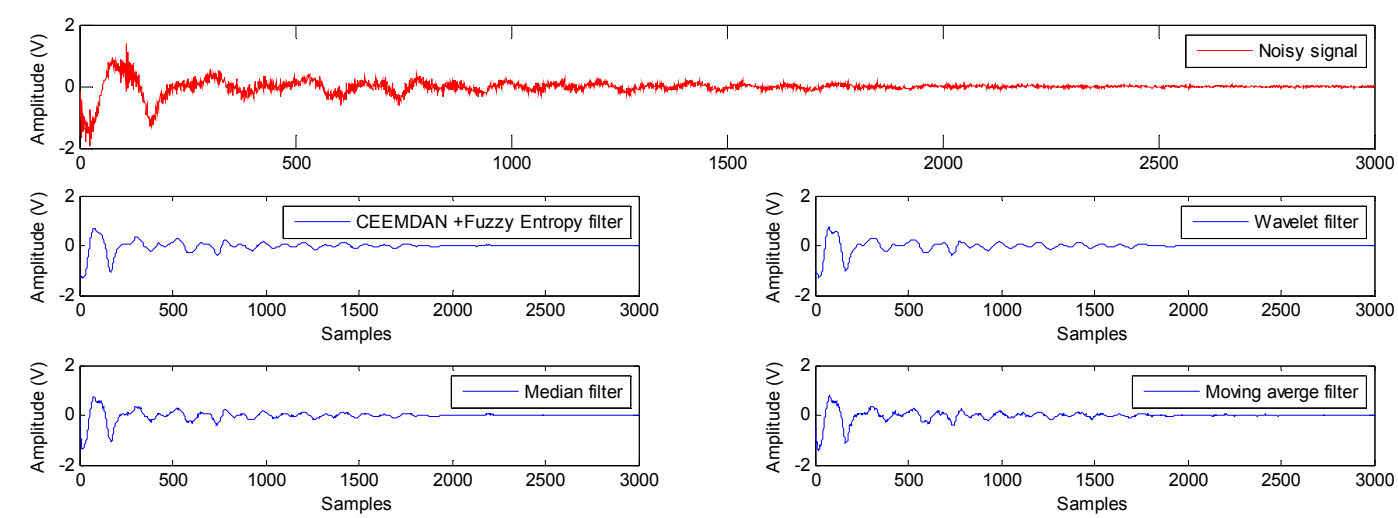

Figure 10. The measured signal and the filtering results of the four filtering methods.

De-trended fluctuation analysis (DFA) is widely used to measure the smoothness of time series. Reference [33] indicates that the larger value of the fractal scaling index $(\alpha)$ in the DFA algorithm, the smoother the time series. To quantify the filtering result, the DFA was used to measure the filtering effect for the above four filtering methods. The fractal scaling index $(\alpha)$ value for each method is in Figure 11. It shows that the $\alpha$ value of the filtering method based on the CEEMDAN and fuzzy entropy is larger than the other three filtering methods. It also illustrates that the performance of the proposed filtering method worked best among the other filtering methods.
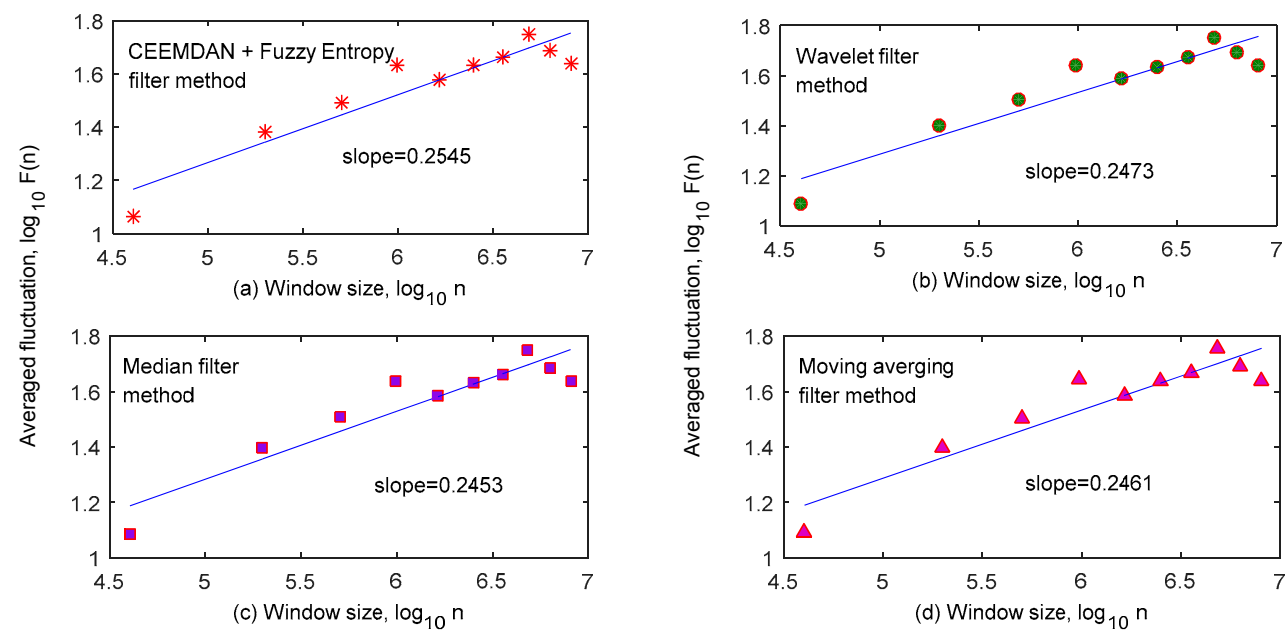

Figure 11. The fractal scaling index of the four filtering methods. (a) CEEMDAN + fuzzy entropy filter method; (b) Wavelet filter method; (c) Median filter method; (d) Moving averging filter method.

In addition, when checking the definition of the mean square error (MSE) and output signal-to-noise ratio $\left(\mathrm{SNR}_{\text {out }}\right)$, it is found that the original signal $\mathrm{y}(\mathrm{n})$ is unknown in Equations (19) and (20), which is often used to evaluate the filtering effectiveness. Now, the noisy signal and filtered signal are known for the measured signal. To evaluate the filtering effectiveness, the original signal $y(n)$ in Equations (19) and (20) is replaced with noisy signal, these equations being re-defined as RMSE and RSNR out$_{\text {, respectively. It is obvious that the evaluated result is opposite to MSE and SNR }}$ out. That means that the larger (smaller) the RMSE (RSNR ${ }_{\text {out }}$ ) is, the better the filtering result [27]. Here, the RSNR ${ }_{\text {out }}$ and RMSE are introduced to evaluate the filtering performance. Table 3 shows 
the values of RSNR out $_{\text {and }}$ RMSE. To be easily observed, the fractal scaling index $(\alpha)$ is also in Table 3 . From Table 3, it is found that the RSNR, RMSE, and the fractal scaling index $(\alpha)$ of the filtering method based on CEEMDAN and fuzzy entropy are smallest, largest, and largest respectively. The result shows that the filtering method based on CEEMDAN and fuzzy entropy had the best performance in removing noise.

Table 3. Values of revised mean squared error (RMSE), revised output signal-to-noise ratio (RSNR ${ }_{\text {out }}$ ), and fractal scaling index for different filtering methods.

\begin{tabular}{cccc}
\hline Filter Method & RSNR $_{\text {out }}$ & RMSE & Fractal Scaling Index $(\boldsymbol{\alpha})$ \\
\hline CEEMDAN & 7.5352 & 0.0086 & 0.2578 \\
Wavelet & 7.9124 & 0.0082 & 0.2473 \\
Median & 8.5065 & 0.0075 & 0.2453 \\
Moving Averaging & 8.2266 & 0.0080 & 0.2461 \\
\hline
\end{tabular}

\section{Conclusions}

In this paper, a novel filtering method was proposed to filter out simulated signal and the impact signal using the CEEMDAN and fuzzy entropy. For the simulated signal filtering, the CEEDAN and fuzzy entropy, CEEMDAN and sample entropy, EEMD and fuzzy entropy, and EEMD and sample entropy are compared to filter simulation signal with different input signal to noise ratio statistically. When SNR and MSE are used to measure the filtering, it shows that the filtering method based on CEEMDAN and fuzzy entropy work best. For the impact signal, three kinds of evaluated criteria for filtering effect are introduced (RSNR, RMSE, and DFA). The result shows that the proposed filtering method outperforms the others in removing noise. From the simulated signal and measured signal, the proposed filtering method (CEEMDAN and fuzzy entropy) is more suitable to filter than the other filtering methods.

Acknowledgments: The authors are grateful for comments and suggestions by anonymous reviewers and the Associate Editor for their valuable comments to prove the quality of the paper significantly.

Author Contributions: Liwei Zhan conceived and designed the experiments; Liwei Zhan performed the experiments; Liwei Zhan and Chengwei $\mathrm{Li}$ analyzed the data; Chengwei Li contributed materials and analysis tools; Liwei Zhan wrote the paper. All authors have read and approved the final manuscript.

Conflicts of Interest: The authors declare no conflict of interest.

\section{References}

1. Ciang, C.C.; Lee, J.R.; Bang, H.J. Structural health monitooring for a wind turbine system: A review of damage detection methods. Meas. Sci. Technol. 2008, 19, 310-314. [CrossRef]

2. Feraboli, P.; Ireland, D.; Kedward, K. The Role of Force and Energy in Low Velocity Impact Events. In Proceedings of the 45th AIAA/AMSE/ASCE/AHS/ASC Structures, Structural Dynamics \& Materials Conference, Palm Spring, CA, USA, 19-22 April 2004.

3. Savadori, A. Impact testing of plastics: Present knowledge. Polym. Test. 1985, 5, 209-241. [CrossRef]

4. Vandergheynst, P.; Antoine, J.P.; Vyve, E.V.; Goldberg, A.; Doghri, I. Modeling and simulation of an impact test using wavelets, analytical solutions and finite elements. Int. J. Solids Struct. 2001, 38, 5481-5508. [CrossRef]

5. Liu, Y.; Liaw, B. Drop-weight impact tests and finite element modeling of cast acrylic/aluminum plates. Polym. Test. 2009, 28, 808-823. [CrossRef]

6. Hazizan, M.A.; Cantwell, W.J. The low velocity impact response of an aluminium honeycomb sandwich structure. Compos. B Eng. 2003, 34, 679-687. [CrossRef]

7. Proakis, J.G.; Mnaolakis, D.G. Digital Signal Processing: Principles, Algorithms, and Applications; Prentice-Hall: Englewood Cliffs, NJ, USA, 1996; pp. 223-229.

8. Goldstein, J.S.; Reed, I.S.; Scharf, L.L. A multistage representation of the Wiener filter based on orthogonal projections. IEEE Trans. Inf. Theory 1998, 44, 2943-2959. [CrossRef] 
9. Strela, V.; Heller, P.N.; Strang, G.; Topiwala, P.; Heil, C. The application of multiwavelet filterbanks to image processing. IEEE Trans. Image Process. 1999, 8, 548-563. [CrossRef] [PubMed]

10. Donoho, D.L. De-noising by soft-thresholding. IEEE Trans. Inf. Theory 1995, 41, 613-627. [CrossRef]

11. Huang, N.E. The empirical mode decomposition and the Hilbert spectrum for nonlinear and non-stationary time series analysis. Proc. R. Soc. Lond. A Math. Phys. Eng. Sci. 1998, 454, 903-905. [CrossRef]

12. Flandrin, P.; Rilling, G.; Goncalves, P. Emperical Mode Decomposition as a filter bank. IEEE Signal Process. Lett. 2004, 11, 112-114. [CrossRef]

13. Ayenu-Prah, A.; Attohokine, N. A criterion for selecting relevant intrinsic mode functions in empirical mode decomposition. Adv. Adapt. Data Anal. 2010, 2, 1-24. [CrossRef]

14. Peng, Z.K.; Tse, P.W.; Chu, F.L. A comparison study of improved Hilbert-Huang transform and wavelet transform: Application to fault diagnosis for rolling bearing. Mech. Syst. Signal Process. 2005, 19, 974-988. [CrossRef]

15. Boudraa, A.O.; Cexus, J.C. EMD-Based signal filtering. IEEE Trans. Instrum. Meas. 2007, 56, $2196-2202$. [CrossRef]

16. Komaty, A.; Boudraa, A.O.; Augier, B.; Daré-Emzivat, D. EMD-based filtering using similarity measure between probability density functions of IMFs. IEEE Trans. Instrum. Meas. 2014, 63, 27-34. [CrossRef]

17. Yang, G.; Liu, Y.; Wang, Y.; Zhu, Z. EMD interval thresholding denoising based on similarity measure to select relevant modes. Signal Process. 2015, 109, 95-109. [CrossRef]

18. Kopsinis, Y.; Mclaughlin, S. Development of EMD-Based Denoising Methods Inspired by Wavelet Thresholding. IEEE Trans. Signal Process. 2009, 57, 1351-1362. [CrossRef]

19. Mert, A.; Akan, A. Detrended fluctuation thresholding for empirical mode decomposition based denoising. Digit. Signal Process. 2014, 32, 48-56. [CrossRef]

20. Siwal, D.; Suyal, V.; Prasad, A.; Mandal, S.; Singh, R. A new approach of denoising the regular and chaotic signals using Empirical Mode Decomposition: Comparison and application. Rev. Sci. Instrum. 2013, 84, 075117. [CrossRef] [PubMed]

21. Wu, Z.; Huang, N.E. Ensemble Empirical Mode Decomposition: A Noise-Assisted Data Analysis Method. Adv. Adapt. Data Anal. 2009, 1, 1-41. [CrossRef]

22. Chan, J.C.; Ma, H.; Saha, T.K.; Ekanayake, C. Self-adaptive partial discharge signal de-noising based on ensemble empirical mode decomposition and automatic morphological thresholding. IEEE Trans. Dielectr. Electr. Insul. 2014, 21, 294-303. [CrossRef]

23. Fang, Y.-M.; Feng, H.-L.; Li, J.; Li, G.-H. Stress Wave Signal Denoising Using Ensemble Empirical Mode Decomposition and an Instantaneous Half Period Model. Sensors 2011, 11, 7554-7567. [CrossRef] [PubMed]

24. Torres, M.E.; Colominas, M.A.; Schlotthauer, G.; Flandrin, P. A complete ensemble empirical mode decomposition with adaptive noise. In Proceedings of the IEEE International Conference on Acoustics, Speech and Signal Process, Prague, Czech Republic, 22-27 May 2011.

25. Humeau-Heurtier, A.; Abraham, P.; Mahe, G. Analysis of laser speckle contrast images variability using a novel empirical mode decomposition: Comparison of results with laser doppler flowmetry signals variability. IEEE Trans. Med. Imaging 2015, 34, 618-627. [CrossRef] [PubMed]

26. Ye, R.; Suganthan, P.N.; Srikanth, N. A Comparative Study of Empirical Mode Decomposition-Based Short-Term Wind Speed Forecasting Methods. IEEE Trans. Sustain. Energy 2015, 6, 236-244.

27. Li, C.; Zhan, L. A hybrid filtering method based on a novel empirical mode decomposition for friction signals. Meas. Sci. Technol. 2015, 26, 125003. [CrossRef]

28. Li, C.; Zhan, L.; Shen, L. Friction Signal Denoising Using Complete Ensemble EMD with Adaptive Noise and Mutual Information. Entropy 2015, 17, 5965-5979. [CrossRef]

29. Pincus, S.M. Approximate entropy as a measure of system complexity. Proc. Natl. Acad. Sci. USA 1991, 88, 2297-2301. [CrossRef] [PubMed]

30. Richman, J.S.; Moorman, J.R. Physiological time-series analysis using approximate entropy and sample entropy. Am. J. Physiol. Heart Circ. Physiol. 2000, 278, H2039-H2049. [PubMed]

31. Chen, W.; Wang, Z.; Xie, H.; Yu, W. Characterization of surface EMG signal based on fuzzy entropy. IEEE Trans. Neural Syst. Rehabil. Eng. 2007, 15, 266-272. [CrossRef] [PubMed] 
32. Zhao, L.; Wei, S.; Zhang, C.; Zhang, Y.; Jiang, X.; Liu, F.; Liu, C. Determination of Sample Entropy and Fuzzy Measure Entropy Parameters for Distinguishing Congestive Heart Failure from Normal Sinus Rhythm Subjects. Entropy 2015, 17, 6270-6288. [CrossRef]

33. Leistedt, S.; Dumont, M.; Lanquart, J.P.; Jurysta, F.; Linkowski, P. Characterization of the sleep EEG in acutely depressed men using detrended fluctuation analysis. Clin. Neurophysiol. 2007, 118, 940-950. [CrossRef] [PubMed]

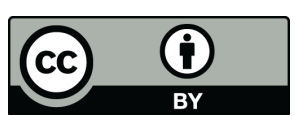

(C) 2016 by the authors; licensee MDPI, Basel, Switzerland. This article is an open access article distributed under the terms and conditions of the Creative Commons Attribution (CC-BY) license (http:// creativecommons.org/licenses/by/4.0/). 